\title{
Locking of multiple resonant mode structures in the reversed-field pinch
}

\author{
A. K. Hansen, A. F. Almagri, D. J. Den Hartog, S. C. Prager, and J. S. Sarff \\ Department of Physics, University of Wisconsin-Madison, 1150 University Avenue, \\ Madison, Wisconsin 53706
}

(Received 16 February 1998; accepted 7 May 1998)

\begin{abstract}
Locking of a rotating mode by applying a resonant magnetic perturbation having the same helicity has been observed on various devices. Experiments have been carried out on the Madison Symmetric Torus reversed-field pinch (RFP) [Dexter et al., Fusion Technol. 19, 131 (1991)] which show that an externally applied magnetic perturbation can cause locking of the dominant magnetic modes (poloidal mode number $m=1$, toroidal mode numbers $n=5-10$ ) when the perturbation is resonant with them. A perturbation which is not resonant $(m=0$ or 2$)$ produces no such effect. Thus, resonant torques may lock a stochastic magnetic structure arising from several modes, as likely exists in the RFP, as well as a distinct island as exists in tokamaks, although the details of the interaction are likely to be different. (C) 1998 American Institute of Physics.
\end{abstract}

[S1070-664X(98)01908-9]

\section{INTRODUCTION}

In the context of magnetic confinement devices such as tokamaks and reversed-field pinches (RFPs), the term "mode locking" is used when a magnetic structure within the plasma becomes stationary with respect to the device wall or an external magnetic perturbation (a "field error"). An example of such a structure is the magnetic island formed by a tearing mode. Mode locking is an issue of ongoing theoretical and experimental work. Besides the issues of deleterious effects on confinement and device operation, mode locking represents interesting plasma physics in its own right.

It has been experimentally demonstrated on various tokamak devices that an applied magnetic perturbation can lock a mode that has the same helicity as the perturbation. For examples see Refs. 1 and 2. The results were consistent with the resonant torque model: the mode with the same helicity as the perturbation locked to it.

A model that is commonly used to understand mode locking is that of a magnetic island in a rotating bulk plasma, the kinematics of which are governed by the balance between the inertia of the island and the electromagnetic and viscous torques that act upon it. ${ }^{3,4}$ The electromagnetic torque is produced by the $\mathbf{J} \times \mathbf{B}$ force between the mode and other magnetic structures, e.g., a stationary field error. The viscous torque is produced by differential rotation between the bulk plasma and the island. One important element of the theory is that a magnetic perturbation must be resonant with the island in order to produce a net torque on it.

In some respects, RFPs and tokamaks are similar. Both can have rotating mode structures present; so the general theoretical framework discussed above for resonant mode locking is applicable to both configurations. There are also significant differences between the two configurations. A tokamak plasma normally has at most one or two active modes, whereas in a reversed-field pinch there are always several dominant active modes. These are long-wavelength, global, tearing modes. The most dominant of these has toroidal mode number $n \approx 2 R / a$, with $R$ and $a$ being the major and minor radii, respectively. For the Madison Symmetric Torus (MST) RFP, ${ }^{5} R=1.5 \mathrm{~m}$, and $a \approx 0.5 \mathrm{~m}$, so the $n=6$ mode is the largest. These modes are typically phase-locked, ${ }^{6}$ and their island structures overlap, as shown in Fig. 1. This overlap of the islands leads to stochastization of the magnetic field throughout much of the plasma. There are also electromagnetic torques being generated by interactions between individual modes via three-wave coupling, e.g., between those with poloidal mode number $m=1$ and the $m=0$ modes which are resonant at the reversal $(q=0)$ radius. ${ }^{7}$ This coupling has been measured bispectrally in MST. ${ }^{8}$

The purpose of the experiments reported here is to test the applicability of the resonant torque theory to the RFP. In particular, the questions which we wished to answer were whether resonant perturbations produce locking of a possibly stochastic structure in the same manner as for distinct magnetic islands, and, if so, whether the effect is altered by the presence of several coupled modes.

The experiments that will be discussed in this paper were performed in the MST RFP. Perturbations which were resonant with the dominant $(m=1)$ modes as well as perturbations which were not resonant with the dominant modes were applied, and the effect on the mode rotation was observed. The perturbations which were applied had $m=0,1$, or 2. The results are consistent with resonant torque theory. The dominant $m=1$ modes were locked by the resonant $(m=1)$ peturbation, and they were not influenced by those which were not resonant with them $(m=0,2)$.

\section{OVERVIEW OF EXPERIMENT}

The procedure that was followed consistently for all configurations was to apply the error field during a discharge with an established rotating mode structure. The diffusion time of MST's aluminum shell is much longer than the 


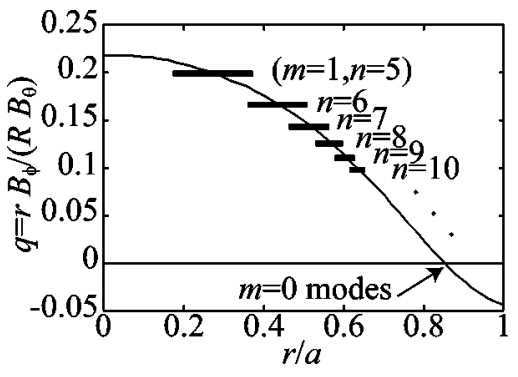

FIG. 1. Typical $q$ profile for MST plasmas, showing island widths for $m$ $=1$ modes.

length of a typical discharge ( $\sim 50 \mathrm{~ms})$; thus external coils would not be effective. Since it is not feasible to place coils inside the vessel, it was necessary to apply the magnetic perturbation through the insulated poloidal (vertical) cut in the shell.

A set of eight coils which are normally used to correct the radial field error at the poloidal cut were co-opted to apply magnetic perturbations with $m=0,1$, or 2 . Because the magnetic perturbations are applied through the poloidal cut, which is about $1 \mathrm{~cm}$ wide, they are approximately spatial delta functions in toroidal angle. Thus, the $n$ spectrum produced is essentially constant for the $n$ 's of interest. This is an adequate configuration for use on MST, because the RFP $q$ profile entails that the broadband (in $n$ ) perturbation applied through the cut can couple with several toroidal modes for each $m$. In all cases, the pulse was applied at $20 \mathrm{~ms}$ into the discharge, which is well within the flattop phase of the plasma current.

The main diagnostics used were internal arrays of magnetic pickup coils. A poloidal array of 16 equally spaced radial pickup coils at the poloidal cut were used to resolve the $m$ spectrum of the radial magnetic field at the cut, including both the applied perturbation and that which was generated by the plasma. A schematic overview of the cut and the pick up coils can be seen in Fig. 2. A poloidal array of 8 and a toroidal array of 32 equally spaced poloidal magnetic field pickup coils were used to resolve the $n$ and $m$ spectra, respectively, of the global modes.

\section{RESULTS OF EXPERIMENT}

The dynamics of the dominant modes in MST are characterized by sudden changes in amplitude and rotation velocity that occurs through a sawtooth oscillation (Fig. 3). Fig-

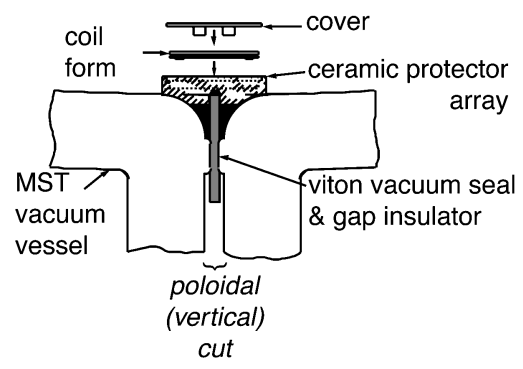

FIG. 2. Detail of the poloidal cut on MST, showing the position of a magnetic pickup coil.

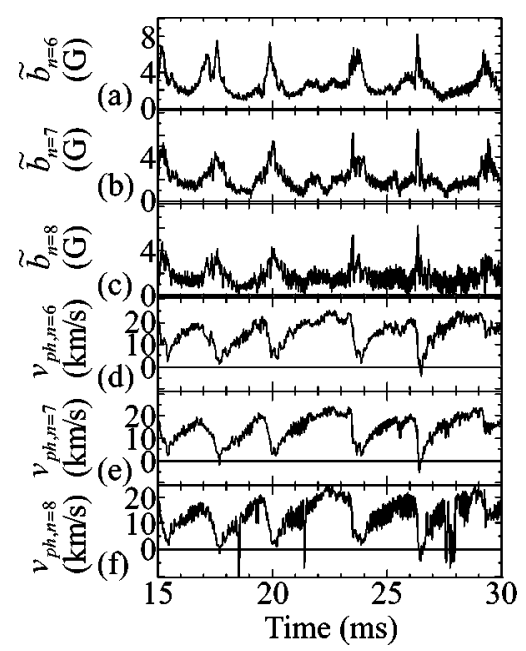

FIG. 3. Discharge with no applied magnetic perturbation. (a)-(c) Dominant MST $m=1$ tearing mode amplitudes, (a) $n=6$, (b) $n=7$, (c) $n=8$. (d)-(f) Toroidal phase velocity of dominant modes (d) $n=6$, (e) $n=7$, (f) $n=8$. The absence of permanent locking is indicated by the continued acceleration and deceleration of the modes. Sawtooth crashes can be seen in the mode deceleration and the mode amplitudes.

ures 3(a)-3(c) show the amplitudes of the $m=1, n=6,7$, and 8 modes, respectively. The $n=6$ mode clearly has the largest amplitude. All three of the mode amplitudes show periods of increased activity. These are sawtooth oscillations, which are characterized by a slow $(\sim 1 \mathrm{~ms})$ phase, and a rapid ( $\sim 100 \mu \mathrm{s})$, or relaxation ("crash") phase. The elevation in amplitude occurs around the time of the crash phase. Figures 3(d)-3(f) show the toroidal phase velocities of the $n=6,7$ and 8 modes, $\left(\nu_{\mathrm{ph}, n}=\omega R / n\right)$. The decelerations and accelerations that can be seen are a normal feature of MST discharges, and they are not strongly dependent on the field error. The three velocities obviously have similar behavior and magnitudes, which reflects the phase locking of the dominant modes. Henceforth, we will use the $n=6$ mode to represent all of the $m=1$ modes.

In the experimental setup which was used, there are field errors at the poloidal cut in MST even in the absence of applied perturbations, as can be seen in Fig. 4. Figures 4(a)4(c) show the $m=0,1$, and 2 components of the radial field at the poloidal cut. These may be taken to be the background upon which we add additional magnetic perturbations. The monotonic increase which can be seen in the $m=2$ component of the field error at the poloidal cut [Fig. 4(c)] is generated by the magnetic field around the (square) poloidal field transformer soaking through the conducting shell. Figures. 4(d) and 4(e) show the $n=6$ toroidal phase velocity and amplitude, respectively. The $m=1$ component of the field error is sufficiently small that the dominant modes are not locked, but are able to rotate freely. The other components of the field error are not resonant with the $m=1$ modes, so they should not affect the $m=1$ mode rotation. The signals from Fig. 4 will be shown in identical format for all the applied magnetic perturbation configurations.

When an $m=1$ magnetic perturbation of sufficient amplitude is applied, the discharge locks. An example is shown in Fig. 5. Figure 5(b), the $m=1$ component of the radial 


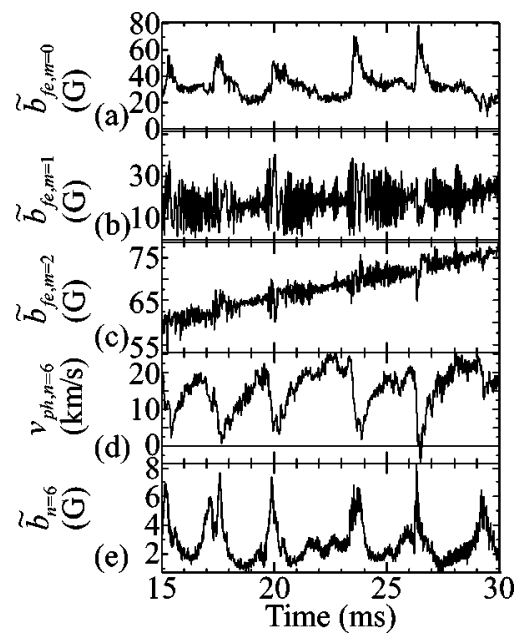

FIG. 4. Discharge with no applied magnetic perturbation. (a)-(c) Poloidal mode components of field error on MST, (a) $m=0$, (b) $m=1$, (c) $m=2$. (d) Toroidal phase velocity of the $n=6$ mode. (e) Amplitude of the $n=6$ mode. The absence of permanent locking is again evident from the continued mode acceleration and deceleration.

magnetic field at the cut, shows the pulsed magnetic perturbation as a large rise from the background level beginning at $20 \mathrm{~ms}$. The spectral purity of the pulse is evidenced by Figs. 5 (a) and 5(c), the $m=0$ and $m=2$ components of the radial field, which show no response (cf. Fig. 4). Locking occurs at $\sim 22 \mathrm{~ms}$ into the discharge, as defined by the velocity of the $n=6$ mode [Fig. 5(d)], which slows to zero and remains stationary thereafter. This locking occurs between the sawtooth crashes, the positions of which can be seen in Fig. 5(e), the $n=6$ mode amplitude. Locking occurs when the $m=1$ component of the radial field at the poloidal cut is about 75 $\mathrm{G}$, or $\sim 7.6 \%$ of the average poloidal field. For reference, the fraction of this radial field that contributes to any individual $n$ is about $1 / 1000$, so the overall $(m=1, n=6)$ perturbation required for locking is about $10^{-4}$ of the average poloidal

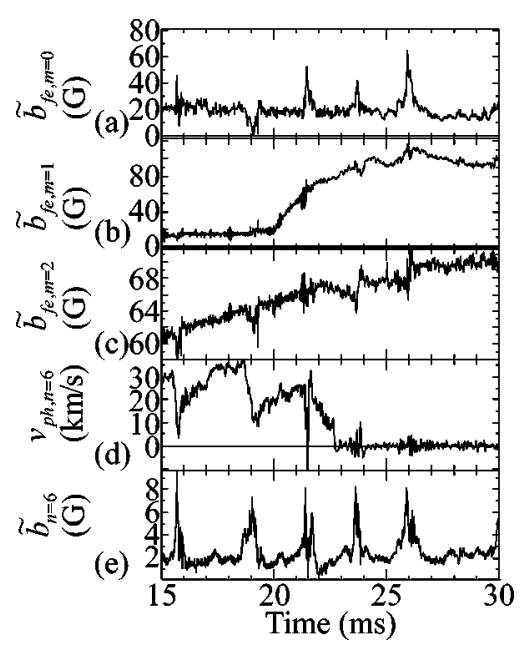

FIG. 5. Discharge with applied $m=1$ magnetic perturbation. (a) -(c) Poloidal mode components of field error on MST, (a) $m=0$, (b) $m=1$, (c) $m$ $=2$. (d) Toroidal phase velocity of the $n=6$ mode. (e) Amplitude of the $n$ $=6$ mode. Permanent locking occurs at around $22 \mathrm{~ms}$, where the mode rotation velocity is zero.

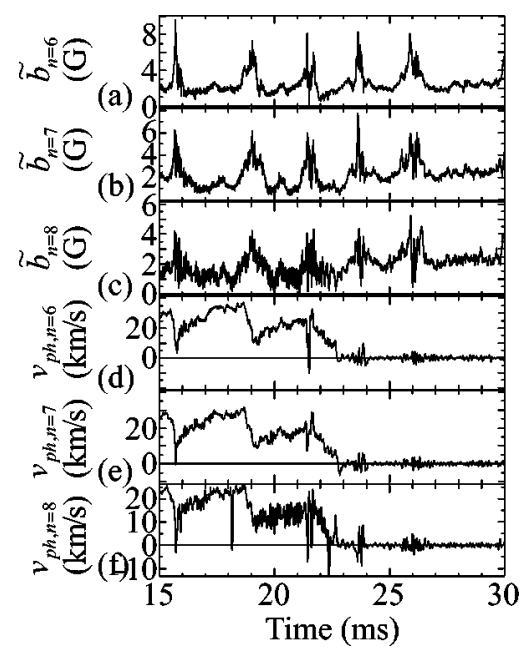

FIG. 6. Discharge with applied $m=1$ magnetic perturbation. (a)-(c) Dominant MST $m=1$ tearing mode amplitudes. (a) $n=6$, (b) $n=7$, (c) $n=8$. (d)-(f) Toroidal phase velocity of dominant modes (d) $n=6$, (e) $n=7$, (f) $n=8$. Locking occurs at $22 \mathrm{~ms}$. The baseline (between sawtooth crashes) mode amplitudes increase after locking, and the phase velocities exhibit similar dynamics.

field. Figure 6 shows that the baseline $n=6,7$, and 8 mode amplitudes increase after locking [Figs. 6(a)-6(c), also cf. Fig. 3], and that all three modes exhibit similar dynamic responses to the field error [Figs. 6(d)-6(f)].

An applied $m=0$ magnetic perturbation produces no effect on the $m=1, n=6$ mode, as can be seen in Fig. 7. This is as expected for a perturbation which is not resonant with the $m=1$ modes. In this case the amplitude of the $m=0 \mathrm{com}-$ ponent of the radial magnetic field at the poloidal cut at a sawtooth crash can reach $90 \mathrm{G}$-comparable to the $m=1$ error needed to induce locking. The amplitude of the global $(m=0, n=1)$ mode increases in response to the perturbation, as shown in Fig. 8. The toroidal phase velocity of the $(m$ $=0, n=1$ ) mode is normally zero in MST, i.e., it can there-

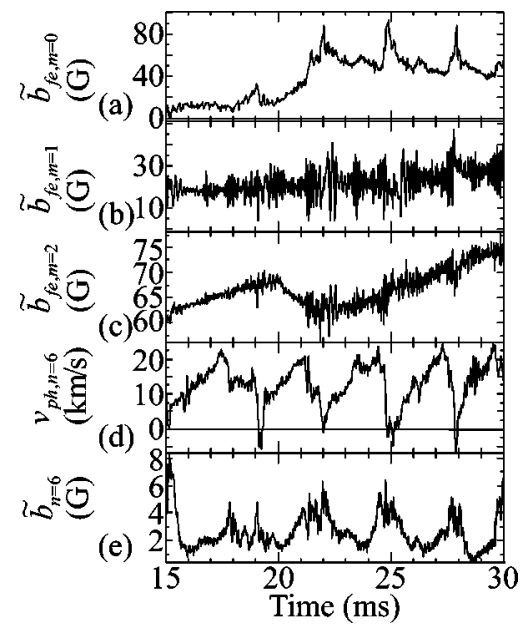

FIG. 7. Discharge with applied $m=0$ magnetic perturbation. (a)-(c) Poloidal mode components of field error on MST, (a) $m=0$, (b) $m=1$, (c) $m$ $=2$. (d) Toroidal phase velocity of the $n=6$ mode. (e) Amplitude of the $n=6$ mode. The $n=6$ mode rotation continues unaffected by the applied error. The decrease in the $m=2$ component of the error is due to an impure applied spectrum. 


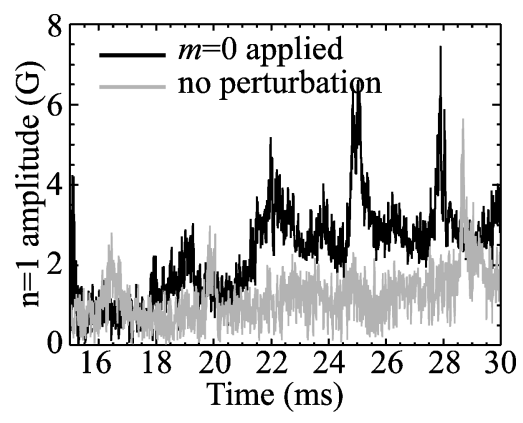

FIG. 8. Comparison of $n=1$ mode amplitudes between discharges with and without applied $m=0$ magnetic perturbation. A large increase is seen in the case when the error is applied.

fore be considered to be a permanently locked mode. The reduction in the $m=2$ amplitude [Fig. 8(c)] is due to the fact that this perturbation was generated using only eight coils and had a significant $m=2$ component along with the dominant $m=0$.

As expected, an applied $m=2$ magnetic perturbation produces no effect on the $m=1, n=6$ rotation, as can be seen in Fig. 9. This holds although the total $m=2$ amplitude of the radial magnetic field at the poloidal cut reaches $190 \mathrm{G}$ (about $20 \%$ of the average poloidal field), i.e., more than twice the amplitude for which locking occurred with an $m$ $=1$ perturbation. An obvious question is whether an $m=2$ perturbation will lock a preexisting $m=2$ mode. In MST, however, the $m=2$ fluctuation occurs with the same frequency and phase as the $m=1$. This can be seen in Fig. 10, where the poloidal rotation frequencies of the $m=1$ and $m$ $=2$ modes have been calculated. Most of the observed $m$ $=2$ fluctuation presumably results from toroidal distortion of the $m=1$, as first reported for the High Beta Toroidal Experiment (HBTX-1A) RFP. ${ }^{9}$ The close correspondence in frequency which can be seen in Fig. 10 suggests that the independent $m=2$ fluctuations are small in MST.

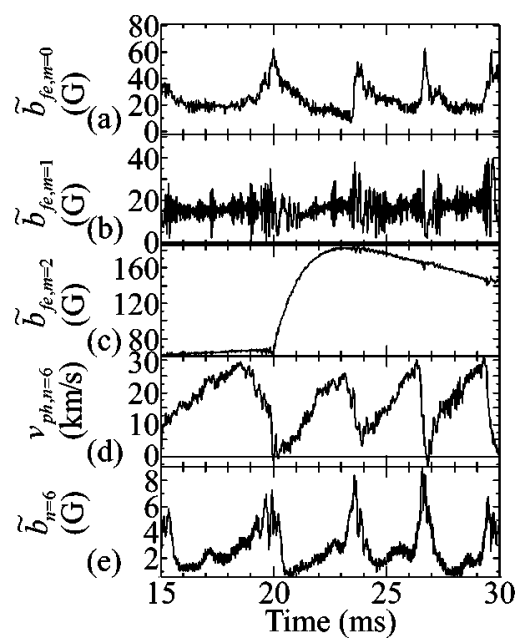

FIG. 9. Discharge with applied $m=2$ magnetic perturbation. (a)-(c) Poloidal mode components of field error on MST, (a) $m=0$, (b) $m=1$, (c) $m$ $=2$. (d) Toroidal phase velocity of the $n=6$ mode. (e) Amplitude of the $n=6$ mode. The $n=6$ mode rotation continues unaffected by the applied error.

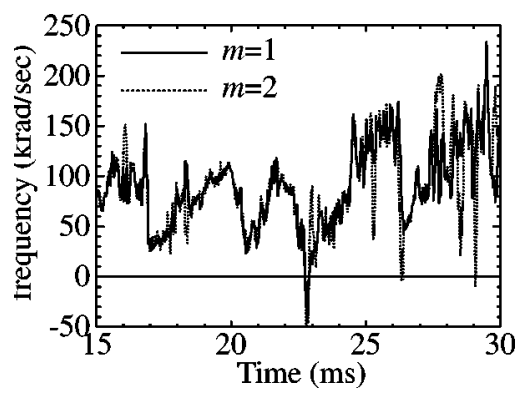

FIG. 10. $m=1$ and $m=2$ rotation frequencies. The frequencies are essentially identical, except for the increased noise in the $m=2$.

\section{DISCUSSION}

That an $m=1$ perturbation can lock the discharge is consistent with the existence of resonant electromagnetic torques between the perturbation and the dominant $(m=1, n$ $=5-10)$ internal modes. This result is consistent with the results from tokamaks. The locking threshold is defined as the condition where the electromagnetic and viscous torques balance one another.

The magnitude of the electromagnetic torque density between the applied error field and a particular (single) island is given by

$$
T_{\mathrm{em}}=\left|\mathbf{R} \times \widetilde{\mathbf{J}}_{\mathrm{fe}} \times \widetilde{\mathbf{B}}\right|,
$$

where $\widetilde{\mathbf{J}}_{\mathrm{fe}}$ is the plasma current induced by the magnetic perturbation, and $\widetilde{B}$ is the amplitude of the pre-existing mode. We will estimate this quantity for an $\mathrm{m}=1$ error field acting on the $(1,6)$ mode from experimental data and physically reasonable scale lengths. These quantities will be evaluated during the steady-state phase (between sawtooth crashes) prior to the mode deceleration [Fig. 5(d)]. Let

$$
\widetilde{J}_{\mathrm{fe}} \equiv \frac{1}{\mu_{0}}\left|\nabla \times \widetilde{\mathbf{B}}_{\mathrm{fe}}\right| \cong \frac{\widetilde{B}_{\mathrm{fe}}}{\mu_{0} W_{1,6}},
$$

be a reasonable estimate, with $\widetilde{B}_{\text {fe }} \approx 1.0 \times 10^{-5} \mathrm{~T}$ - the $(1,6)$ component of a $1.0 \times 10^{-2} \mathrm{~T} m=1$ field error at the poloidal cut - and $W_{1,6}$ the width of the $(1,6)$ island $(\sim 0.08 \mathrm{~m})$. Thus $\widetilde{J}_{\mathrm{fe}} \approx 100 \mathrm{~A} / \mathrm{m}^{2}$. With $R \approx 1.5 \mathrm{~m}$, i.e., the major radius and $\widetilde{B} \approx 2.0 \times 10^{-4} \mathrm{~T}$, the measured amplitude of the $(1,6)$ mode, the electromagnetic torque density $T_{\mathrm{em}} \approx 3.0 \times 10^{-2} \mathrm{~N} / \mathrm{m}^{2}$.

The magnitude of the viscous torque density at the rational sur face is given by

$$
T_{V s} \approx \mu_{\perp} R \frac{\partial\left(\Delta \Omega_{s}\right)}{\partial r},
$$

where the subscript " $s$ ", refers to the value at the rational surface, $\mu_{\perp}$ is the perpendicular viscosity, and $\Delta \Omega_{s}$ is the difference between the "natural" rotation frequency of the island and the surrounding plasma. We will use $\Delta \Omega_{s} \approx 2$ $\times 10^{4} \mathrm{~s}^{-1}$, the measured difference between the $n=6$ mode and plasma angular velocities. ${ }^{10}$ The measured value of the global perpendicular momentum diffusion coefficient in MST is $\nu_{\perp} \approx 55 \mathrm{~m}^{2} / \mathrm{s}$. ${ }^{11}$ This figure may not reflect the local viscosity between a magnetic island and the plasma outside the island, but we will use it as a first approximation. The 
viscosity is related to the diffusion coefficient by $\mu_{\perp}$ $=\rho \nu_{\perp}$, where $\rho$ is the mass density. At a number density of $1 \times 10^{19} \mathrm{~m}^{-3}$ in a hydrogen plasma, $\rho$ is $1.67 \times 10^{-8} \mathrm{~kg} / \mathrm{m}^{3}$, so $\mu_{\perp} \approx 9.2 \times 10^{-7} \mathrm{~kg} / \mathrm{m} / \mathrm{s}$. If we now let

$$
\frac{\partial\left(\Delta \Omega_{s}\right)}{\partial r} \approx \frac{\Delta \Omega_{s}}{W_{1,6}}=2.5 \times 10^{5} \mathrm{~m}^{-1} \mathrm{~s}^{-1},
$$

and use $R=1.5 \mathrm{~m}$, we find $T_{V s} \approx 0.345 \mathrm{~N} / \mathrm{m}^{2}$. The agreement between electromagnetic and viscous torques is not particularly close in this case. However, this picture is probably too simple, because we have neglected the role of interactions between the islands.

As a better estimate for this more complicated system, instead of a single mode, we will estimate the torque balance on the group of modes which are phase-locked together in the stochastic region extending out roughly to the $q=0$ surface. By considering the almost rigid-rotor behavior of this group of modes (cf. Fig. 3), the forces acting between islands are implicitly included. Since the islands overlap, the electromagnetic torque density in the stochastic region formed by the $n=5-10$ modes is like some multiple of the value calculated above for the single $n=6$ mode, perhaps twice as large, so we estimate $T_{\mathrm{em}} \approx 2 \times 0.03 \mathrm{~N} / \mathrm{m}^{2}=0.06 \mathrm{~N} / \mathrm{m}^{2}$. To estimate the viscous torque density, we use the same global viscosity as above, noting that it is now more appropriate given the larger volume of plasma included. For the scale length of the velocity gradient, we use the $q=0$ radius, $\sim 40$ $\mathrm{cm}$, as a reasonable estimate considering the relatively flat velocity profile implied by the similarity of the $n=6,7$, and 8 phase velocities shown in Fig. 3. For the same measured $\Delta \Omega_{s}$ as above, we find

$$
\frac{\partial\left(\Delta \Omega_{s}\right)}{\partial r} \approx \frac{\Delta \Omega_{s}}{r_{q=0}}=5.0 \times 10^{4} \mathrm{~m}^{-1} \mathrm{~s}^{-1},
$$

or $T_{V s} \approx 0.069 \mathrm{~N} / \mathrm{m}^{2}$. The agreement between the electromagnetic and viscous torques in this case is much better than in the prior estimate. The viscous torque density is less in this case than for a single island, but that is not altogether surprising, since we are encompassing a larger volume, while the viscosity is presumably a surface effect.

\section{SUMMARY AND CONCLUSIONS}

We have applied magnetic perturbations having poloidal mode number $m=0,1$ and 2 . Only the $m=1$ configuration, which is resonant with the dominant modes in MST, induced locking of those modes. The $m=0$ configuration did produce an increase in the amplitude of the $(m=0, n=1)$ mode, but this mode is already locked in MST discharges. There is, however the possibility of a nonlinear three-wave interaction like that between $(m=0, n=1),(m=1, n),(m=1, n \pm 1)$, which could affect the $m=1$ modes. The global $n=1$ mode does indeed respond to the perturbation, however, no effect is seen on the $m=1$ modes. We conclude that the amplitude of the $(m=0, n=1)$ is not yet large enough for the threewave interaction to lock the $m=1$ modes. The $m=2$ configuration did not lock the $m=1$ modes, either. The independent $m=2$ modes are probably not large enough to show the effects of an applied $m=2$ perturbation.

Thus, it appears that the rudimentary concepts of mode locking via resonant torques, formulated for rotating islands, may also apply to the situation in which distinct islands do not exist. That is, a stochastic structure, generated by several modes, may also experience resonant torques. Future experiments will focus on independent control of the rotation of the individual modes, and, perhaps, the stochasticity caused by the modes.

\section{ACKNOWLEDGMENT}

This work was supported by the U.S. Department of Energy.

${ }^{1}$ J. T. Scoville, R. J. LaHaye, A. G. Kellman, T. H. Osborne, R. D. Stambaugh, E. J. Strait, and T. S. Taylor, Nucl. Fusion 31, 875 (1991).

${ }^{2}$ T. C. Hender, R. Fitzpatrick, A. W. Morris, P. G. Carolan, R. D. Durst, T. Edlington, J. Ferreira, S. J. Fielding, P. S. Haynes, J. Hugill, I. J. Jenkins, R. J. LaHaye, B. J. Parham, D. C. Robinson, T. N. Todd, M. Valovic, and G. Vayakis, Nucl. Fusion 32, 2091 (1992).

${ }^{3}$ R. Fitzpatrick, Nucl. Fusion 33, 1049 (1993).

${ }^{4}$ R. Fitzpatrick, R. J. Hastie, T. J. Martin, and C. M. Roach, Nucl. Fusion 33, 1533 (1993).

${ }^{5}$ R. N. Dexter, D. W. Kerst, T. W. Lovell, S. C. Prager, and J. C. Sprott, Fusion Technol. 19, 131 (1991).

${ }^{6}$ A. F. Almagri, S. Assadi, S. C. Prager, J. S. Sarff, and D. W. Kerst, Phys. Fluids B 4, 4080 (1992).

${ }^{7}$ C. C. Hegna, Phys. Plasmas 3, 4646 (1996).

${ }^{8}$ S. Assadi, S. C. Prager, and K. L. Sidikman, Phys. Rev. Lett. 69, 281 (1992).

${ }^{9}$ I. H. Hutchinson, M. Malacarne, P. Noonan, and D. Brotherton-Ratcliffe, Nucl. Fusion 24, 59 (1984).

${ }^{10}$ D. J. Den Hartog, A. F. Almagri, J. T. Chapman, H. Ji, S. C. Prager, and J. S. Sarff, Phys. Plasmas 2, 2281 (1995).

${ }^{11}$ A. F. Almagri, J. T. Chapman, C.-S. Chiang, D. J. Den Hartog, G. Fiksel, S. C. Prager, J. S. Sarff, M. R. Stoneking, and D. J. Woehrer, Bull. Am. Phys. Soc. 41, 1410 (1996). 